\title{
INSAR SENSITIVITY ANALYSIS OF TANDEM-L MISSION FOR MODELING VOLCANIC AND SEISMIC DEFORMATION SOURCES
}

\author{
Homa Ansari $^{(1,2)}$, Kanika Goel ${ }^{(1)}$, Alessandro Parizzi ${ }^{(1)}$, Henriette Sudhaus $^{(3)}$, Nico Adam ${ }^{(1)}$, Michael Eineder ${ }^{(1,2)}$ \\ ${ }^{(1)}$ Remote Sensing Technology Institute (IMF), German Aerospace Center (DLR), Email: homa.ansari@dlr.de \\ ${ }^{(2)}$ Chair of Remote Sensing Technology (LMF), Technical University of Munich (TUM), Munich, Germany \\ ${ }^{(3)}$ Institute of Earth and Environmental Science, University of Potsdam
}

\begin{abstract}
We assess the reliability of space-borne InSAR-derived deformation source parameters for volcanic and seismic events, with special focus on the future L-band data of the proposed Tandem-L mission [1-3]. Using representative simulation cases, the influence of certain characteristics of the InSAR measurements on the source model parameter precision is quantified. The performance drivers are assessed from two aspects: the data acquisition geometry as well as the measurement noise; in particular governed by signal coherence and superposed atmospheric signal. The significance of each these governing noise components is shown to be dependent on the spatial scale of the geophysical signal of interest as well as the deformation source mechanism in question. Here, we estimate the error bounds for the inferred source parameters as a function of the signal coherence and atmospheric signal parameters.
\end{abstract}

\section{INTRODUCTION}

Interferometric Synthetic Aperture Radar (InSAR) technique has been widely used for monitoring the Earth deformation as well as modeling the sources causing such deformation. Although a great number of publications have focused on the application of InSAR in source modeling and development of inversion algorithms, much less attention has been dedicated to the reliability of InSAR in source modeling. The aim of this paper is to introduce the InSAR performance drivers and quantify their influence on reliability of the InSAR derived source parameters. With special focus dedicated to the performance of the German Aerospace Center's (DLR) future L band SAR mission, Tandem-L [1-3].

The reliability of source modeling is in close relation with the data quality, processing algorithms, complexity of the model and the deformation magnitude caused by the source. Therefore a comprehensive reliability analysis shall cover all these aspects. Being focused on the InSAR performance, our analysis is mostly dedicated to the investigation of the data quality. We therefore constrain the other aspects by consideration of volumetric deformation sources, such as volcanic events, and crustal dislocation sources, such as seismic events. We further limit the analysis to a few representative cases with the purpose to introduce estimated error bounds for source modeling of the mentioned events.

The main aim of this work is thus on understanding the propagation of InSAR data errors to the source parameters and of quantifying the effect of InSAR limiting factors on the precision of derived source parameters.

\section{DEFORMATION SOURCE MODELING}

The crustal deformation is captured by differential InSAR measurements, as a projection of the 3D surface displacement in the Line of Sight (LOS) direction of the sensor [4]:

$$
d_{L O S}=\left(d_{n} \cdot \sin \alpha-d_{e} \cos \alpha\right) \sin \theta+d_{u} \cdot \cos \theta
$$

Where $d_{e}, d_{n}, d_{u}$ are the displacement components in the local east, north and up direction; and $\alpha$ and $\theta$ are the heading angle of the satellite and incidence angle of the SAR beam, respectively.

The observed surface displacement can be related to the geophysical processes causing the three-dimensional surface deformation through a model $(G)$ of a set of parameters $(\boldsymbol{x})$ :

$$
d=\boldsymbol{e} \cdot G(\boldsymbol{x})
$$

With $\boldsymbol{e}$ as the unit vector which projects the threedimensional modelled displacement into the LOS direction.

Inverting Eq. 2, the InSAR measurements may be used to infer the geophysical parameters of the deformation source.

The focus of this work is on modelling the seismic and volcanic activities; for which the simple and commonly used Okada dislocation model [5] and the Mogi point- 
source model [6] in the elastic half-space are introduced as the relevant $G(\boldsymbol{x})$. In this way, without loss of generality, we simplify volcanic sources with point sources and seismic ruptures by uniform slip on a single rectangular fault.

The point-source Mogi model is considered for radialsymmetric deformation in an elastic half-space caused by volume change inside a spherical magma chamber. The model is described by four model parameters: three geometric parameters for the horizontal location as well as the depth of the magma chamber and the fourth accounting for the volume change inside the chamber.

The elastic half-space Okada model relates the surface displacement to a rectangular fault plane with a uniform slip. The fault plane is described by its location, orientation and extension. The location is conventionally given by the east, north and depth coordinates of the fault centre. The orientation is given with respect to the north direction and horizontal surface with the strike and dip angles, respectively. The extension is defined by the width and length of the rectangular plane. The magnitude of the fault relative displacement during the rupture is defined by the slip parameter and the fault slip mechanism is given by the rake, as the direction of the slip on the fault plane.

\section{InSAR PERFORMANCE DRIVERS}

\subsection{SAR Geometrical Limitations}

Projecting the displacement in the LOS direction; the InSAR measurements are limited in capturing the 3D displacement field. The special choice of near-polar sun- synchronous orbits of typical SAR satellites causes a poor sensitivity of the InSAR measurements to the displacement in south-north direction [7].

Dependent on the geometry of the deformation source, the poor insensitivity to the north motion component poses problems for precise unambiguous source modeling. To improve the sensitivity and modelling accuracy the fusion of multi-aspect InSAR measurements from different ascending/descendingright/left- looking acquisitions and/or fusion of the pixel tracking techniques in the azimuth is suggested $[7,8]$. Pending is the quantification of the performance gain for the multi-aspect fusion.

\subsection{InSAR Measurements' Noise}

Inspired by [4], we distinguish between two different stochastic mechanisms in modeling InSAR noise. The first category contains the error sources affecting the single InSAR measurements referred to as decorrelation sources; the second category accounts for the spatial correlation between the single measurements.

\section{Single Point Decorrelation}

Decorrelation noise is defined here as the error sources with correlation length small enough to introduce no covariance between the measurements. Examples can be the induced noise by the SAR instrument, processing algorithms, temporal and volumetric scattering mechanisms, etc. The total phase coherence of a single pixel is then derived as [9]:

$$
\gamma_{\mathrm{tot}}=\prod_{i} \gamma_{i}
$$

In which $\gamma_{i}$ corresponds to each of the decorrelation sources. The phase standard deviation relation to the total coherence is approximated by [10]:

$$
\sigma_{\phi}^{2}=\frac{1-\gamma_{\mathrm{tot}^{2}}}{2 N \gamma_{\mathrm{tot}^{2}}}
$$

with $N$ as the number of multi-looked pixels. Given the SAR wavelength $\lambda$, the phase dispersion is propagated to the maximum achievable precision of the LOS deformations:

$$
\sigma_{d_{L O S}}^{2}=\left(\frac{\lambda}{4 \pi}\right)^{2} \cdot \sigma_{\phi}^{2}
$$

Thus the single points' stochastic model reads as:

$$
Q_{\text {s.p. }}=\sigma_{d_{L O S}}^{2} \cdot I
$$

with $I$ introduced as an identity matrix.

\section{Spatial Correlation}

Spatially propagating over the interferogram, the second type of error sources introduces correlation between the single measurements. The errors induced by imprecise satellite orbit, low resolution DEM, phase unwrapping algorithms and atmospheric wave propagation effects are categorized in this group.

We treat the errors related to satellite orbits and utilized DEM as deterministic errors which may be modeled and compensated; therefore neglect them in the stochastic model. We also exclude the phase unwrapping errors as their impact is beyond the scope of this study. The remaining source of spatially correlated error is therefore the atmospheric wave propagation effect also known as the Atmospheric Phase Screen (APS).

The atmospheric effects are divided into dispersive and non-dispersive parts, caused by the ionospheric and tropospheric layers, respectively. The dispersive delay is dependent on the SAR wavelength and expected to be mitigated to a large extent by multi-spectral approaches [11], the residual errors maybe considered in the stochastic model. 
The covariance function of the residual tropospheric delay has been well studied and a number of models have been suggested in the literature [e.g. 4, 12]. In order to generalize this stochastic model, two simplifications are considered. Firstly, we assume a flat area with no tropospheric stratification. Secondly, the tropospheric signal is considered to be stationary and isotropic; thus it is only a function of the distance between the measurement points. Under these assumptions, the Matern-family model [12, 13] has been chosen to describe the covariance function of the delay:

$$
q_{i, j}=\sigma_{T P S}^{2} \cdot \frac{1}{2^{v-1} \cdot \Gamma(v)} \cdot\left(\frac{2 \sqrt{v}|d|}{\rho}\right)^{v} \cdot K_{v}\left(\frac{2 \sqrt{v}|d|}{\rho}\right)
$$

Defined by the following functions/parameters:

\section{$\Gamma($.$) : Gamma function$}

$K_{v}($.$) : modified Bessel function of the second kind$ $\sigma_{T P S}^{2}$ : variance of the residual tropospheric delay

$v$ : smoothness parameter

$\rho: \quad$ tropospheric correlation length

$d$ : distance between single measurements $i, j$

Based on the covariance function, the covariance matrix of observations is obtained:

$$
Q_{\mathrm{APS}}=\left[\begin{array}{cccc}
q_{1,1} & q_{1,2} & \ldots & q_{1, n} \\
q_{2,1} & q_{2,2} & \ldots & q_{2, n} \\
\vdots & \vdots & \ddots & \vdots \\
q_{n, 1} & q_{n, 2} & \ldots & q_{n, n}
\end{array}\right]
$$

The different driving mechanisms of the single point and APS noises allow for an addition of the two introduced stochastic models [4], such that the joint stochastic model for InSAR measurement reads as:

$$
Q_{\mathrm{d}}=Q_{\text {s.p. }}+Q_{\mathrm{APS}}
$$

\section{METHODOLOGY IN PERFORMANCE ASSESSMENT}

In order to assess the reliability of an inferred deformation source model from the SAR data, the study of the a posteriori probability density function (PDF) of the model parameters is the appropriate statistical tool. The precision of the estimation may be inferred from the marginal PDFs of the single parameters while the joint PDF between parameters give an indication of their mutual attributes such as their correlation.

Here we use the first order reliability methods [14] to estimate the covariance function of the source parameters using:

$$
Q_{x}=J \cdot Q_{d} \cdot J^{T}
$$

with $J$ as the Jacobian matrix of the SAR measurements with respect to the deformation source parameters $x$ :

$$
J=\left[\begin{array}{ccc}
\frac{\partial d_{1}}{\partial x_{1}} & \cdots & \frac{\partial d_{1}}{\partial x_{n}} \\
\vdots & \ddots & \vdots \\
\frac{\partial d_{m}}{\partial x_{1}} & \cdots & \frac{\partial d_{m}}{\partial x_{n}}
\end{array}\right]
$$

$Q_{d}$ as the measurements' covariance matrix defined in section 3.2.3, and $\{\},.\{\}^{T}$ as the matrix multiplication and transpose operation, respectively.

Following this approach two approximations are introduced: first, the a posteriori PDF of the parameters is approximated by a Gaussian function, which does not allow for multi-modality or asymmetry of the error structure. Second, the nonlinear source model is linearized by the first term of its Taylor expansion. The latter effect is minimized by evaluation of Jacobian matrix at the solution point (the global minimum of the cost function) of the model.

The covariance function obtained by Eq. 11 is the initial point of the error assessment; with its diagonal elements approximating the estimation error variance and its $2 \times 2$ submatrices representing the joint bivariate normal PDF between parameter pairs.

\section{PERFORMANCE ANALYSIS}

\subsection{Geometrical Performance Assessment}

The geometrical performance is considered by comparing the reliability of source parameters under three different scenarios: single aspect geometry, multiaspect geometry comprised of two descending and one ascending tracks, all captured in right-looking mode, and finally a combination of ascending/descending tracks from right and left-looking directions (Tab. 1). The first two geometrical scenarios are chosen according to the nominal acquisitions planned for Tandem-L. The left-looking is also added in the third case to quantify the possible gain of this acquisition mode.

The a posteriori model covariance function is obtained by Eq. 11 . The measurement's stochastic model $\left(Q_{d}\right)$ is not included to isolate the pure impact of geometry. The estimated covariance matrices are normalized by the increment in the number of observations from one geometric scenario to the other, so that the obtained results merely indicate the effect of geometry and do not benefit from the increased number of observations in multi-aspect scenarios. The comparisons are considered for Mogi and Okada model, separately. 
Table 1. Geometrical comparison scenario

\begin{tabular}{|lccc|}
\hline Scenario & Heading ang.: $\alpha$ & Incidence ang.: $\theta$ & Mode \\
\hline Single aspect & $-12^{\circ}$ & $43^{\circ}$ & Asc. R. \\
\hline Multi-aspect & $-12^{\circ}$ & $43^{\circ}$ & Asc. R. \\
\cline { 2 - 4 } Right-looking & $-168^{\circ}$ & $43^{\circ}, 23^{\circ}$ & Desc. R. \\
\hline $\begin{array}{l}\text { Multi-aspect } \\
\text { Left/Right }\end{array}$ & $-12^{\circ}$ & $43^{\circ}$ & Asc. R.. \\
\cline { 2 - 4 } looking & $-168^{\circ}$ & $43^{\circ}$ & Desc. R. \\
\cline { 2 - 4 } & $-12^{\circ}$ & $-23^{\circ}$ & Asc. R. \\
\cline { 2 - 4 } & $-168^{\circ}$ & $-23^{\circ}$ & Desc. R. \\
\hline
\end{tabular}

\subsubsection{Volcanic Modelling}

A magma chamber in a depth of $3 \mathrm{~km}$ and with a volume change of $6 \times 10^{6} \mathrm{~m}^{3}$ is assumed in the volcanic deformation simulation. The three geometrical scenarios (Tab. 1) are considered in modelling the corresponding deformation source; each data set is comprised of 3000 measurement points spread randomly over the scene.

Fig. 1 shows the error ellipses as 95\% confidence area of the bivariate normal distribution between each model parameter pair. The figure reveals a strong correlation between the depth and volume change which is common (and well-known) in the modelling of surface displacement data. This correlation is not reduced when using the multi-aspect measurements. In contrast to that, a slight correlation between the horizontal coordinates of the modelled point source decreases with the multiaspect geometry scenario. It can thus be interpreted as an effect of the SAR geometric limitations.

The standard deviation of estimated model parameters obtained for each of the geometrical scenarios is depicted in Fig. 2. Compared to the single aspect scenario, the figure depicts the maximum gain of 6 and 8 percent in multi-aspect right- and right/left- looking scenarios, respectively. The sufficiency of the singleaspect InSAR is evident from this result. In case of the Mogi source-model, it is evident that single geometry measurements suffice for inversion of the model parameters. However, it should be noted that this result is obtained for the simple radial-symmetric models. The situation will be different in scenarios where asymmetric or multi-source models are required for volcanic modelling.

\subsubsection{Seismic Modelling}

With single aspect InSAR measurements being less sensitive to the motion in south-north direction, the seismic modelling is most problematic in cases where the 3D surface displacement has its main component in this direction. The magnitude of the decomposed motion in different directions depends solely on the orientation of the fault and direction of the slip; indicated by the dip and rake parameters of the model. In this regard, two events are considered as the best-case and worst-case
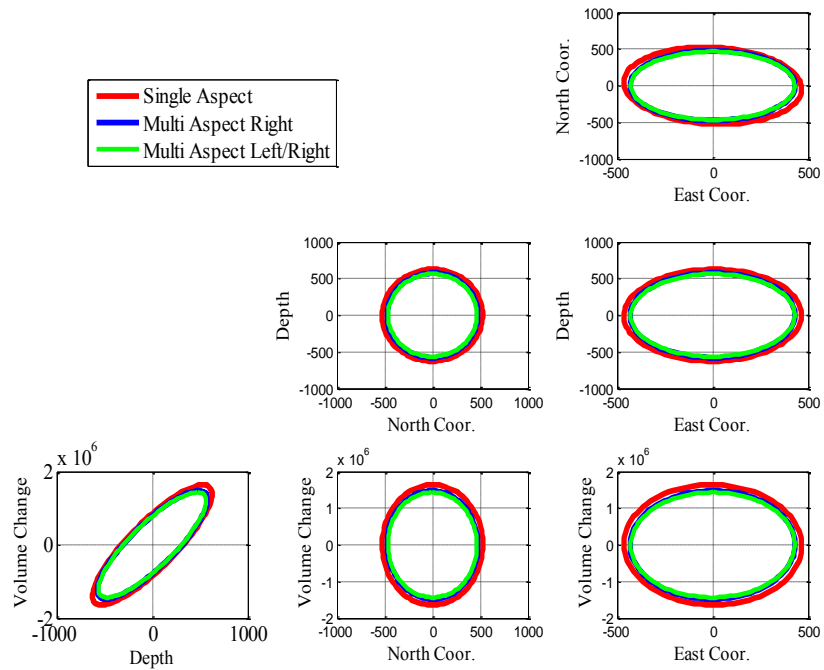

Figure 1. Volcanic model parameter $95 \%$ error ellipses as sections of the bivariate normal distribution of error between the parameter pairs of Mogi model, reported for the three geometric scenarios listed in Tab. 1

geometric scenarios. The former has 20 percent of the
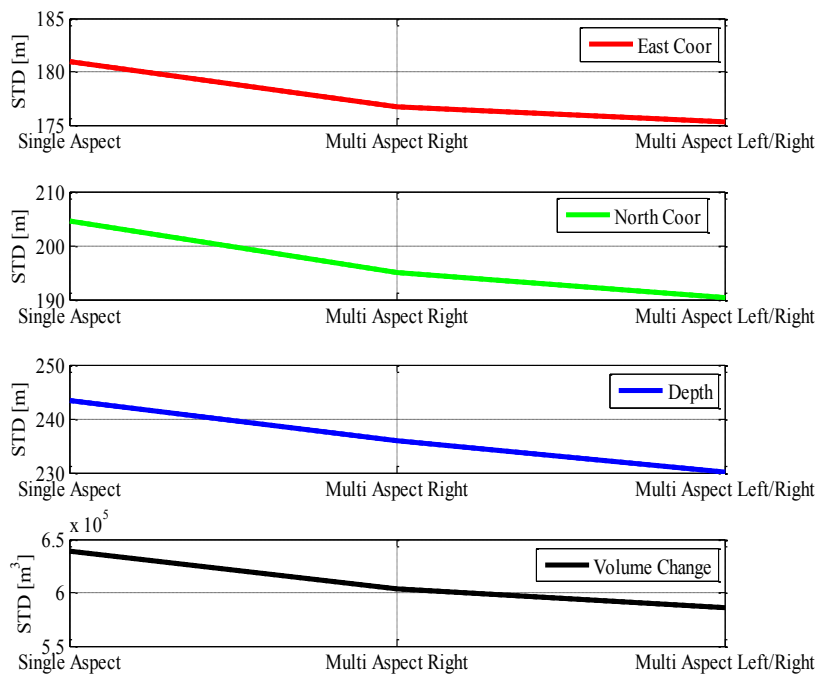

Figure 2. Volcanic model parameters standard deviations normalized by the number of observations, reported for three different geometric scenarios listed in Tab. 1

total deformation in the north component and the latter 70 percent (Fig. 3). The simulated faults are $20 \mathrm{~km}$ long, $11 \mathrm{~km}$ wide and located at $5.5 \mathrm{~km}$ depth. Both are reverse faults $\left(\right.$ rake $=90^{\circ}$ ) and slip by $5 \mathrm{~m}$. The first fault strikes east-west and with dip of 26 degrees, while the second fault strikes north-south with dip of 50 degrees.

Following the comparison strategy of section 5.1.1, the standard deviation of the estimated model parameters under each of the acquisition scenarios of Tab. 1 are 

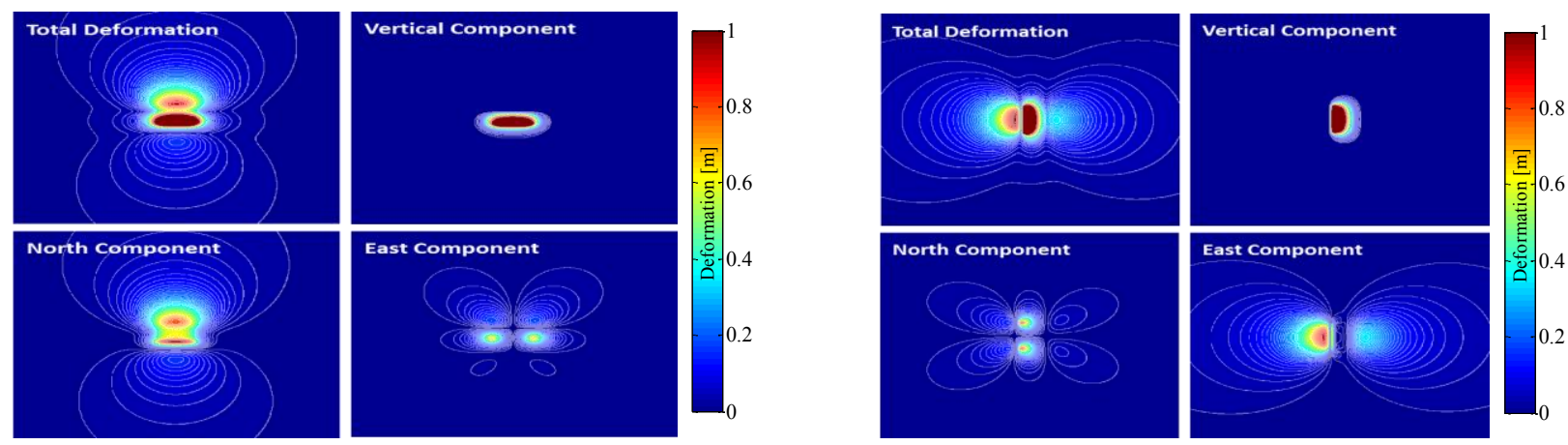

Figure 3. Total and decomposed surface displacement patterns of the simulated faults: Fault 1 (left), Fault 2 (right); with maximum and minimum south-north displacement respectively
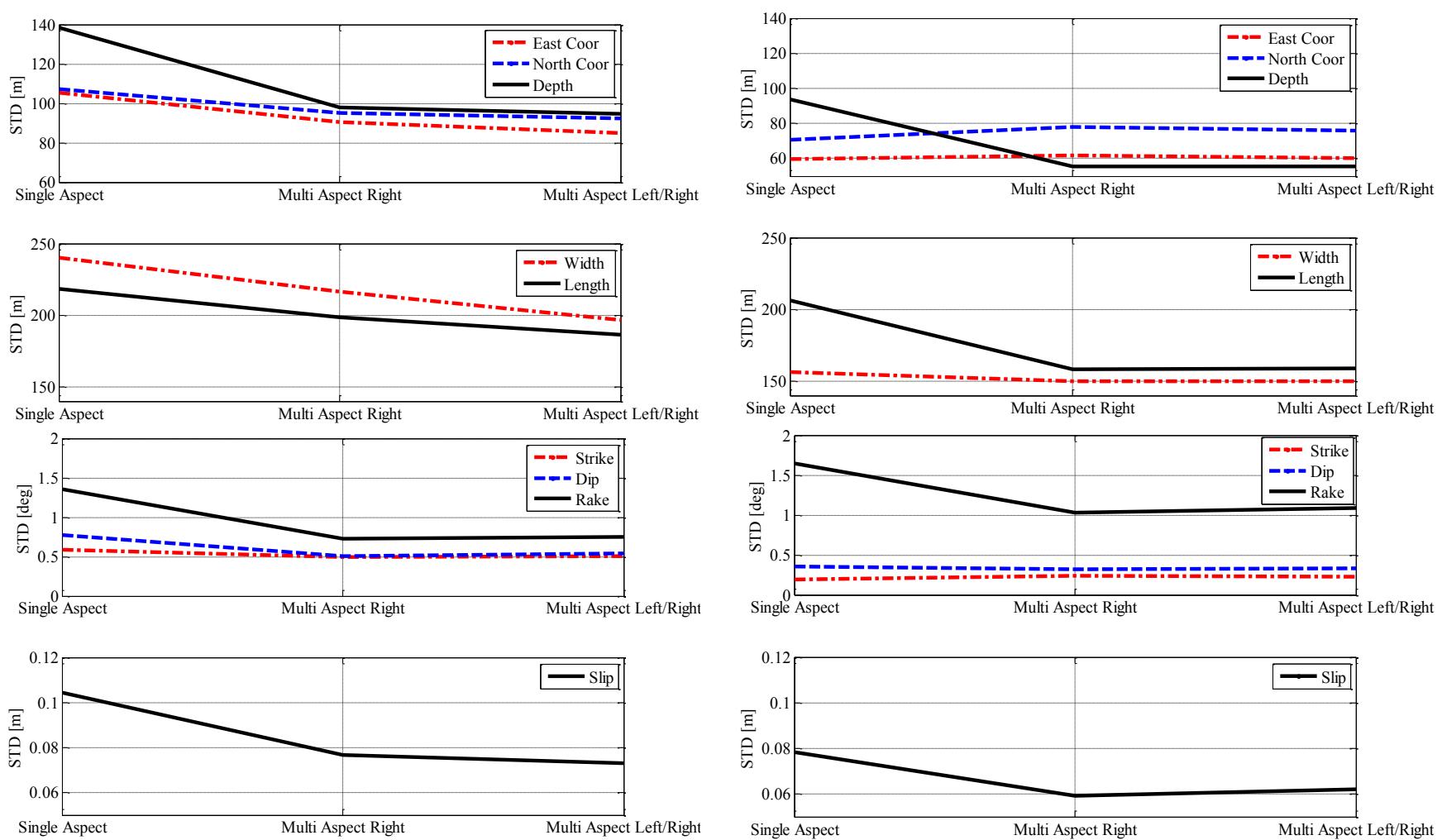

Figure 4. Fault model parameter standard deviations normalized by the number of observations: Fault 1 (left column) and Fault 2 (right column) reported for three different geometric scenarios listed in Tab. 1

analysed (Fig. 4). As expected, the overall modelling precision is higher when the north motion is minimum. The multi-aspect geometry proves to be more beneficial in case of fault 1 , where constraining the north motion with multi-aspect acquisitions is crucial. In this case adding the left-looking geometry further improves the retrieval of the north component, hence the modelling precision. In case of fault 2 , addition of left-looking geometry results in no gain in the parameter estimation.

\subsection{Error Assessment}

As the final part of the assessment, the mentioned measurements' typical error budgets of section 3.2 are considered in the error propagation in order to introduce minimum modelling error bounds. As a nominal acquisition scenario, the multi-aspect right-looking geometry (Tab. 1) is considered in all the analysis of this section. Fixing the geometry, two different governing factors remain for performance analysis: the signal coherence as well as the atmospheric effects. We isolate each of these factors and study their individual impact in source modelling.

The effect of signal coherence is studied by its variation in the relevant interval of $[0,1]$. The atmospheric effects 
are investigated separately by variation of the atmospheric error; in ranges of $[2,40] \mathrm{mm}$, as well as the atmospheric correlation length; in ranges of $[1,5]$ $\mathrm{km}$ (Eq. 9). In all our analysis we also consider the residual errors corresponding to the ionospheric corrections.

In all the comparison cases, 600 measurements spread randomly over the scene are simulated for each acquisition. The estimation window of $50 \mathrm{~m} \times 50 \mathrm{~m}$ is considered for simulation of the InSAR measurements. The SAR resolution is adapted according to Tandem- $\mathrm{L}$ case; with azimuth resolution of $10 \mathrm{~m}$ and range resolution of 85 and $20 \mathrm{MHz}$ (corresponding to maximum 4.5 and $18 \mathrm{~m}$ ground resolution) in ascending and descending tracks, respectively. The comparisons are considered for Mogi and Okada model, separately.

\subsubsection{Volcanic Modelling}

For the analysis of this section the same magma chamber of section 5.1.1, in a depth of $3 \mathrm{~km}$ and with a volume change of $6 \times 10^{6} \mathrm{~m}^{3}$ is assumed in the volcanic deformation simulation.

\section{Average Coherence}

The effect of signal coherence is isolated by fixing the atmospheric signal [atmospheric error $=10 \mathrm{~mm}$, APS correlation length $=1.5 \mathrm{~km}]$. Under this condition, the variation of parameter standard deviation relative to the change in the average coherence is investigated (Fig. 5). As apparent from the figure, for coherence more than 0.5 the gain in modelling precision is negligible.
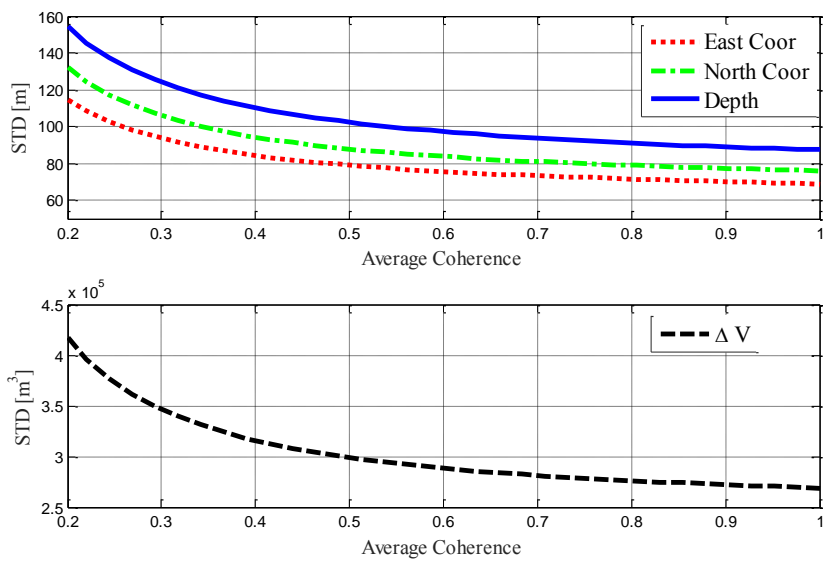

Figure 5. Effect of average coherence on the precision of the estimated model parameters

\section{Atmospheric effect}

The atmospheric impact is investigated in two parts by variation of the atmospheric power (Fig. 6) as well as the atmospheric correlation length (Fig. 7). As a fair assumption for $\mathrm{L}$ band data, the average coherence is fixed to 0.4 in these comparison cases.
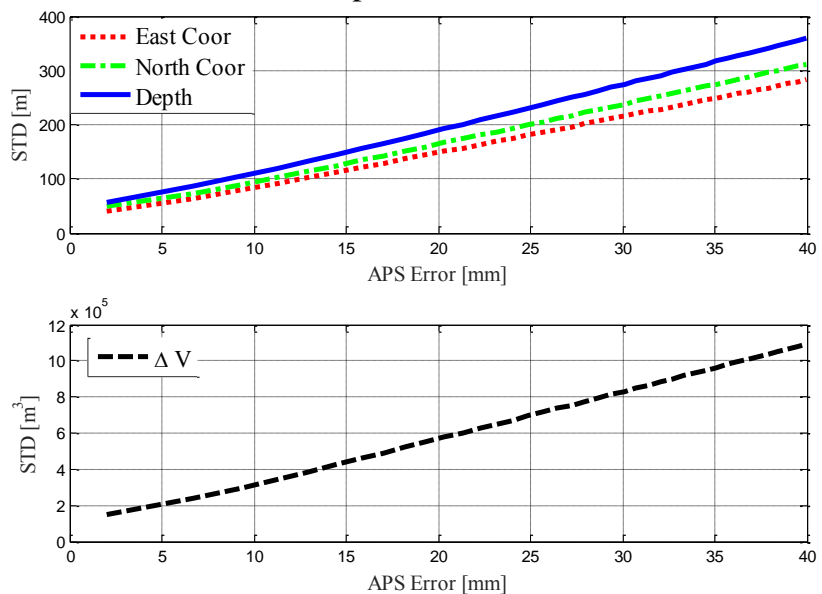

Figure 6. Effect of atmospheric error on precision of the estimated model parameters
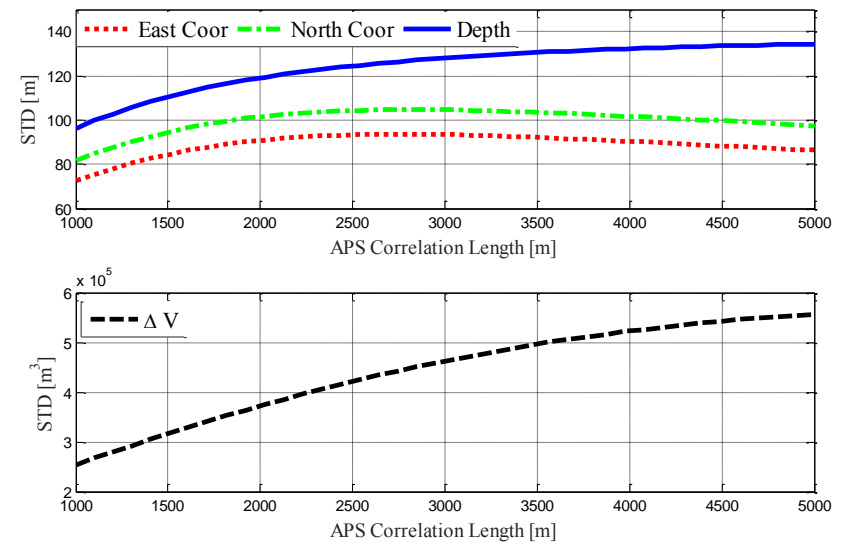

Figure 7. Effect of atmospheric correlation length on the precision of the estimated model parameters; the observed peak in the East and North component indicates a correlation between the spatial scale of deformation signal (related to depth parameter) and the spatial scale of APS

We find a linear trend of increasing model parameter standard deviations with increasing APS error (Fig. 6). In contrast to that, the effect of the APS correlation length is non-linear (Fig. 7). The standard deviation function shows a peak plateau at APS correlation length (2.5 to $3.5 \mathrm{~km})$ around the simulated model depth $(3 \mathrm{~km})$; observed for the east and north coordinates of the point source(Fig. 7). A likely explanation for this peak is a superposition of the geophysical and atmospheric signal with similar spatial correlation length on InSAR measurements. Hence the very similar signals cannot be distinguished and the modelling precision has a minimum. In this case the position of the minimum modelling precision peak would be depending on the spatial wavelength of the displacement signal and thereby on the pointsource depth. 

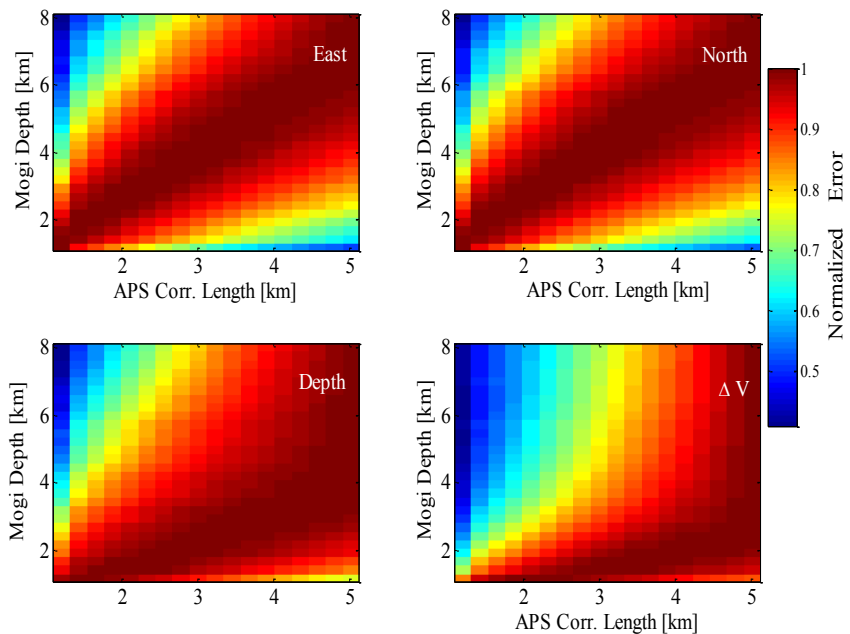

APS Corr. Length $[\mathrm{km}]$

\section{Average Coherence}

The effect of average coherence is isolated by fixing the atmospheric signal [atmospheric error $=10 \mathrm{~mm}$, APS correlation length $=1.5 \mathrm{~km}]$. The impact of coherence on the modelling precision is depicted in Fig. 9.
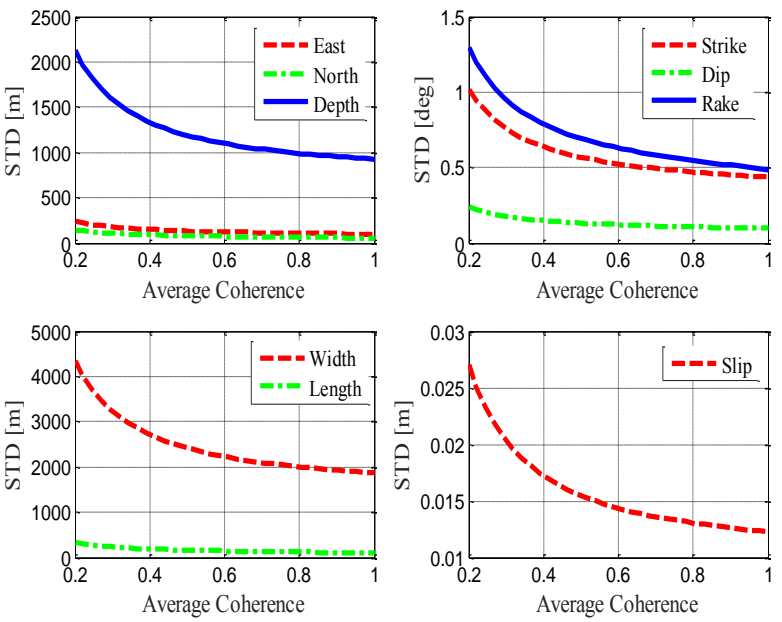

Figure 9. Effect of average coherence on precision of the estimated model parameters volcanic sources with model depth ranging from [1 to 8] $\mathrm{km}$. For each of the simulated cases the modelling precision is investigated for APS correlation length in a range of [1 to 5] km, leading to graphs similar to Fig. 7 for each model depth. In order to capture the location of the peak in each of the resulting graphs, the parameter error is normalized to the maximum error in each depth. The normalized error for each volumetric source is depicted in Fig. 8. The analysis reveals the correlation of the modelling precision for all model parameters with the source depth and the APS correlation lengths. The correlation between source depth and APS correlation length is stronger for the horizontal location model parameters compared to the source depth and source volume change.

\subsubsection{Seismic Modelling}

The detectability and the modelling precision of seismic events depend on their depth and magnitude. In general the shallower and/or higher magnitude events allow a higher modelling accuracy [15]. In order to provide the lower bound errors in seismic modelling, we narrow the analysis down to a medium magnitude/depth event with moment magnitude $M_{w}=6.16$ and in hypocentre depth of $4 \mathrm{~km}$. The fault and rupture orientation are chosen to represent a strike-slip fault, to which the InSAR is less sensitive [15]. The simulated parameters read as: [length, width, depth, strike, dip, rake, slip $]=[10 \mathrm{~km}, 8$ $\left.\mathrm{km}, 4 \mathrm{~km}, 0^{\circ}, 90^{\circ}, 0^{\circ}, 0.7 \mathrm{~m}\right]$. The effect of measurement noise on modelling this simulated event is as follows:

\section{Atmospheric effect}

The variation of the APS error and correlation length is reported by fixing the coherence level to 0.4 . The simulated atmospheric signal is correlated at most in distance of $5 \mathrm{~km}$ while the geophysical signal has spread of tens of kilometres. This trend changes for shallower events with lower magnitude where the spatial scale of defamation signal is low enough to introduce correlation with the atmospheric signal, as seen in the case of Mogi (section 5.2.1).
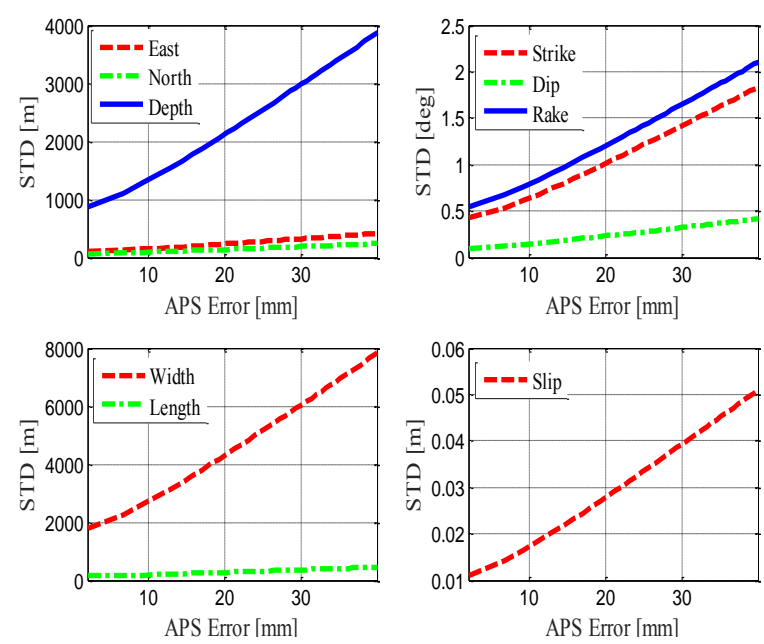

Figure 10. Effect of atmospheric error on precision of the estimated model parameters 

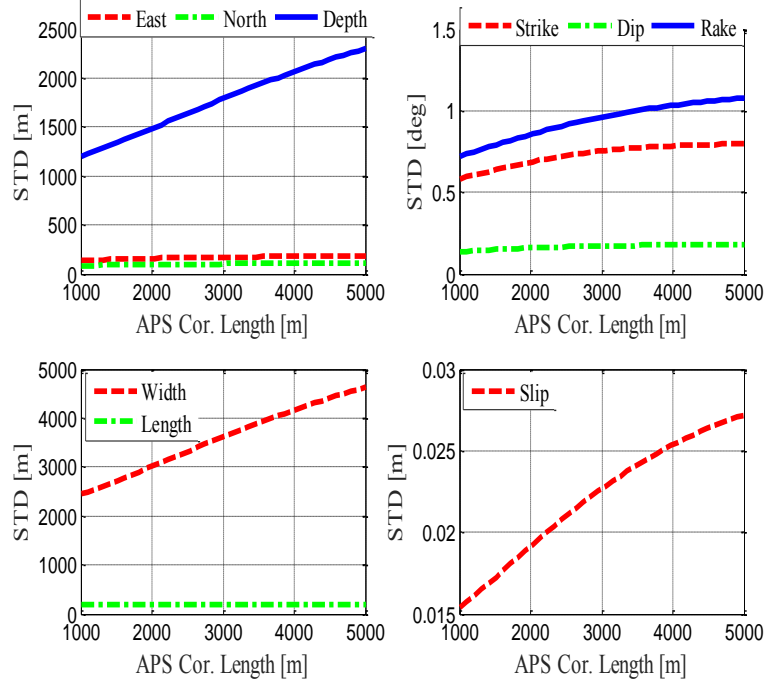

Figure 11. Effect of atmospheric correlation length on precision of the estimated model parameters

\section{CONCLUSIONS}

Using error propagation schemes, we studies the precision of estimated source parameters from InSAR measurements, with special focus on the L band SAR.

Assessing the acquisition geometry reveals the limited performance of SAR in case the major deformation falls in the south-north direction; the direction to which the single aspect InSAR measurements are less sensitive. In such cases the combination of SAR acquisitions from multi aspects assists in improving the modelling performance; with left- and right- looking mode shown to be more efficient than the right-looking.

Investigating the effect of InSAR noise on modelling precision leads to the following results:

- The significance of the decorrelations (investigated by variation in signal coherence) compared to the atmospheric errors depends on the magnitude and spatial scale of the signal

- The modelling accuracy strongly depends on the decoupling of the atmospheric and geophysical signal; in special cases when the spatial scale of the two signals are close, the modelling precision is severely compromised

- The modelling precision of the fault dislocations depends not only on their magnitude and depth but also on the orientation of the fault and the direction of the fault slip

- Deformation magnitude defines the strength of the geophysical signal, trivially the higher the deformation magnitude is the better the modelling precision would be.

\section{REFERENCES}

[1] Krieger, G., Hajnsek, I., Papathanassiou, K., Eineder, M., Younis, M., De Zan, F., Prats, P., Huber, S., Werner, M., Fiedler, H., Freeman, A., Rosen, P., Hensley, S., Johnson, W., Veilleux, L., Grafmueller, B., Werninghaus, R., Bamler, R. \& Moreira, A. (2009). The tandem-L mission proposal: Monitoring earth's dynamics with high resolution SAR interferometry. Radar Conference, 2009 IEEE , vol., no., pp.1,6, 4-8

[2] Krieger, G., Hajnsek, I., Papathanassiou, K., Eineder, M., Younis, M., De Zan, F., Huber, S., Lopez-Dekker, P., Prats, P., Werner, M., Shen, Y., Freeman, A., Rosen, P., Hensley, S., Johnson, W., Veilleux, L., Grafmueller, B., Werninghaus, R., Bamler, R. \& Moreira, A. (2010). Tandem-L: And innovative interferometric and polarimetric SAR mission to monitor earth system dynamics with high resolution. Geoscience and Remote Sensing Symposium (IGARSS), 2010 IEEE International, vol., no., pp. 253,256, 25-30

[3] Shimada, M., Eineder, M., Papathanassiou, K., Motohka, T., Watanabe, M., Ohki, M., De Zan, F., Lopez-Dekker, P., Krieger, G. \& Moreira, A. (2014). Tandem-L: Science Requirements and Mission Concept. EUSAR 2014; 10th European Conference on Synthetic Aperture Radar; Proceedings of, pp. 1-4. VDE, 2014.

[4] Hanssen, R. F. (2001). Radar interferometry: data interpretation and error analysis. Vol. 2. Springer Science \& Business Media.

[5] Okada, Y. (1985). Surface deformation due to shear and tensile faults in a half-space. Bulletin of the seismological society of America 75.4 pp 1135-1154.

[6] Mogi, K. (1958). Relations between the eruptions of various volcanoes and the deformations of the ground surfaces around them.

[7] Wright, T. J., Parsons, B. E., \& Lu, Z. (2004). Toward mapping surface deformation in three dimensions using InSAR. Geophysical Research Letters, 31(1).

[8] Simons, M., Fialko, Y., \& Rivera, L. (2002). Coseismic deformation from the $1999 \mathrm{Mw} 7.1$ Hector Mine, California, earthquake as inferred from InSAR and GPS observations. Bulletin of the Seismological Society of America, 92(4), 1390-1402.

[9] Zebker, H. A., \& Villasenor, J. (1992). Decorrelation in interferometric radar echoes. Geoscience and Remote Sensing, IEEE Transactions on, 30(5), 950-959.

[10] Rodriguez, E., \& Martin, J. M. (1992). Theory and design of interferometric synthetic aperture radars. In Radar and Signal Processing, IEE Proceedings F (Vol. 139, No. 2, pp. 147-159). IET.

[11] Rosen, P. A., Hensley, S., \& Chen, C. (2010). Measurement and mitigation of the ionosphere in L-band interferometric SAR data. In Radar Conference, 2010 IEEE (pp. 1459-1463). IEEE.

[12] Knospe, S., \& Jonsson, S. (2010). Covariance estimation for dInSAR surface deformation measurements in the presence of anisotropic atmospheric noise. Geoscience and Remote Sensing, IEEE Transactions on, 48(4), 2057-2065.

[13] Guttorp, P., \& Gneiting, T. (2006). Studies in the history of probability and statistics XLIX On the Matern correlation family. Biometrika, 93(4), 989-995.

[14] Baecher, G. B. \& John T. Christian. (2005) Reliability and statistics in geotechnical engineering. John Wiley \& Sons.

[15] Dawson, J., \& Tregoning, P. (2007). Uncertainty analysis of earthquake source parameters determined from InSAR: A simulation study. Journal of Geophysical Research: Solid Earth (1978-2012), 112(B9). 